\title{
Implementación de la guía de valoración y manejo del dolor, de la Registered Nurses' Association of Ontario (RNAO) en el programa de Enfermería de la Universidad Autónoma de Bucaramanga
}

Implementation of the Assessment and Pain Management Guidelines of the Registered Nurses' Association of Ontario (RNAO) in the Nursing program of the Autonomous University of Bucaramanga

\section{Implementação do guia de avaliação e gerenciamento de dor da Associação de Enfermeiros Registrados de Ontário (RNAO) no programa de Enfermagem da Universidade Autônoma de Bucaramanga}

\author{
Adriana Patricia Bonilla-Marciales, Enf., Esp., MSc. * \\ Mary Luz Jaimes-Valencia, Enf., MSc., PhD. ** \\ Sergio Eduardo Serrano-Gómez, MD., MSc. ***
}

\section{Resumen}

Introducción: El programa de enfermería de la Universidad Autónoma de Bucaramanga busca innovar con un currículo globalizado que responda a las necesidades tanto del país como del mundo, para ello se ha vinculado con el proyecto International Best Practice Spotlight Organization, para la implementación a nivel curricular de la guía de buenas prácticas clínicas de valoración y manejo del dolor basadas en la evidencia científica de la Registered Nurses' Association

\author{
Gloria Inés Arenas-Luna, Microbiol., Esp., MSc. **** \\ Clara Inés Padilla-García, Enf., Esp., MSc. ***** \\ María Lucenith Criado-Morales, Enf., MSc. ******
}

of Ontario. Objetivo: Reflexionar sobre la experiencia de la implementación de la guía de buenas prácticas clínicas para la valoración y manejo del dolor de la Registered Nurses' Association of Ontario en el programa de Enfermería de la Universidad Autónoma de Bucaramanga. Discusión: Para la metodología de la implementación se consideró como marco de referencia el Proyecto Educativo Institucional, proyecto educativo del programa de la Universidad Autónoma de Bucaramanga, la herramienta de Implementación de buenas prácticas propuesta por la Registered Nurses' Association of

\footnotetext{
Enfermera, especialista en Docencia Universitaria, magíster en Educación con énfasis en Pedagogía, docente Programa de Enfermería, Facultad de Ciencias de la Salud, Universidad Autónoma de Bucaramanga, Floridablanca, Santander, Colombia.

Enfermera, magíster en Enfermería con énfasis en Salud Familiar, doctorado en Enfermería Práctica y Educación, docente programa de Enfermería Facultad de Ciencias de la Salud, Universidad Autónoma de Bucaramanga, Floridablanca, Santander, Colombia.

*** Médico, magíster en Epidemiología, docente programa de Medicina, Facultad de Ciencias de la Salud, Universidad Autónoma de Bucaramanga, Floridablanca, Santander, Colombia.

**** Microbióloga, especialista en Docencia Universitaria, magíster en Microbiología, magíster en Educación, programa de Medicina, Facultad de Ciencias de la Salud, Universidad Autónoma de Bucaramanga, Floridablanca, Santander, Colombia.

***** Enfermera, especialista en Atención de Enfermería en Cuidado Crítico, magíster en Enfermería con énfasis en Cuidado Cardiovascular, docente Escuela de Enfermería, Facultad de Salud, Universidad Industrial de Santander, Bucaramanga, Santander, Colombia.

***** Enfermera, magíster en Enfermería con énfasis en Salud Familiar, docente Programa de Enfermería, Facultad de Ciencias de la Educación y de la Salud, Fundación Universitaria de San Gil - Unisangil, San Gil, Santander, Colombia.
}

Correspondencia: Adriana Patricia Bonilla Marciales. Universidad Autónoma de Bucaramanga, Facultad de Ciencia de la Salud, Programa de Enfermería, Calle 157 No 14-55, Floridablanca, Santander, Colombia. Teléfono+576436111, extensión 555. E-mail: abonilla712@unab.edu.co. 
Ontario y el levantamiento de información que permitiera identificar los vacíos y las acciones de implementación en los contenidos curriculares respecto al uso de recomendaciones de práctica clínica, para la valoración y manejo del dolor en el currículo del programa de enfermería. Conclusiones: Realizar el levantamiento de información de los contenidos curriculares según las recomendaciones permitió identificar los mínimos curriculares a garantizar tales como: mecanismos del dolor, clasificación, intervenciones farmacológicas, no farmacológicas, implicaciones éticas, gestión y fortalecimiento de la actitud profesional que permita brindar un plan de atención integral del ser humano en cada una de las etapas del ciclo vital. La implementación de la guía de buenas prácticas clínicas de valoración y manejo del dolor permitirá tener un impacto académico en la formación de los futuros profesionales del programa de enfermería, una vez se incluyan transversalmente los contenidos relacionados con las buenas prácticas en las diferentes asignaturas del componente básico, profesional y específico del nuevo plan curricular. [Bonilla-Marciales AP, Jaimes-Valencia ML, Serrano-Gomez SE, Arenas-Luna GI, Padilla-García Cl, Criado-Morales ML. Implementación de la guía de valoración y manejo del dolor, de la Registered Nurses' Association of Ontario (RNAO) en el programa de Enfermería de la Universidad Autónoma de Bucaramanga. MedUNAB 2017; 20(2): 148-164].

Palabras clave: Enfermería; Enfermería Basada en la Evidencia; Dolor; Guía de Práctica Clínica; Estudiantes de Enfermería.

\section{Abstract}

Introduction: The nursing program of the Autonomous University of Bucaramanga seeks to innovate with a globalized curriculum that responds to the needs of both the country and the world, to that end, it has been linked to the international project International Best Practice Spotlight Organization, for the implementation at the curricular level of good clinical practices for the assessment and pain management guidelines based on the scientific evidence of the Registered Nurses' Association of Ontario. Objective: To reflect on the experience of the implementation of good clinical practices for the assessment and management of pain guidelines of the Registered Nurses' Association of Ontario in the Nursing program of the Autonomous University of Bucaramanga. Discussion: For the methodology of its implementation, the Institutional Educational Project was considered as a reference framework, educational project of the program of the Autonomous University of Bucaramanga, the implementation tool of good practices proposed by the Registered Nurses' Association of Ontario and the gathering of information that would allow to identify the gaps and the implementation actions in the curricular contents regarding the use of clinical practice recommendations, for the assessment and management of pain in the curriculum of the nursing program. Conclusions: Carrying out the information gathering of the curricular contents according to the recommendations allowed identifying the minimum curricula to be guaranteed such as mechanisms of pain, classification, pharmacological and non-pharmacological interventions, ethical implications, management and strengthening of the professional attitude that allows providing an integral attention program for the human being in each stage of the life cycle. The implementation of good clinical practices of assessment and pain management guidelines will allow an academic impact in the training of future professionals at the nursing program, once the contents related to good practices in the different subjects of the basic, professional and specific component of the new curricular plan are included transversally. [Bonilla-Marciales AP, Jaimes-Valencia ML, Serrano-Gomez SE, Arenas-Luna GI, Padilla-García Cl, Criado-Morales ML. Implementation of the Assessment and Pain Management Guidelines of the Registered Nurses' Association of Ontario (RNAO) in the Nursing Program of the Autonomous University of Bucaramanga. MedUNAB 2017; 20(2): 148-164].

Keywords: Nursing; Evidence-Based Nursing; Pain; Practice Guideline; Students, Nursing.

\section{Resumo}

Introdução: O programa de enfermagem da Universidade Autônoma de Bucaramanga procura inovar com um currículo globalizado que responde às necessidades do país e do mundo, pois isso tem sido associado ao projeto internacional International Best Practice Spotlight Organization, para a implementação no nível curricular do guia de boas práticas clínicas de avaliação e gerenciamento de dor com base na evidência científica da Associação de Enfermeiros Registrados de Ontário. Objetivo: Reflectir sobre a experiência da implementação do guia de boas práticas clínicas para avaliação e manejo da dor de Associação de Enfermeiros Registrados de Ontário no programa de Enfermagem da Universidade Autônoma de Bucaramanga. Discussão: para a metodologia da implementação, o Projeto Educacional Institucional, o projeto de programa educacional da Universidade Autônoma de Bucaramanga, a ferramenta de implementação de boas práticas proposta pelo Associação de Enfermeiros Registrados de Ontário e a coleta de informações que foi considerada como um quadro de referência permitiu identificar as lacunas e as ações na implementação dos conteúdos curriculares sobre o uso das recomendações da prática clínica, para a avaliação e o gerenciamento da dor no currículo do programa de enfermagem. Conclusões: Realização da coleta de informação dos conteúdos curriculares de acordo com as recomendações permitidas para identificar os currículos mínimos a serem garantidos, tais como: mecanismos de dor, classificação, intervenções farmacológicas, implicações nãofarmacológicas, éticas, gerenciamento e fortalecimento da atitude profissional que permita fornecer um plano de atenção integral do ser humano em cada dos estágios do ciclo de vida. A implementação da guia de boas práticas clínicas na avaliação e o gerenciamento de dor permitirá um impacto acadêmico no treinamento dos futuros profissionais do programa de enfermagem, uma vez que os conteúdos relacionados às boas práticas nos diferentes assuntos do programa sejam incluídos transversalmente. Componente básico, profissional e específico do novo programa curricular. [Bonilla-Marciales $A P$, JaimesValencia ML, Serrano-Gomez SE, Arenas-Luna GI, PadillaGarcía Cl, Criado-Morales ML. Implementação do guia de avaliação e gerenciamento de dor da Associação de Enfermeiros Registrados de Ontário (RNAO) no programa de Enfermagem da Universidade Autônoma de Bucaramanga. MedUNAB 2017; 20(2): 148-164].

Palavras-chave: Enfermagem; Enfermagem Baseada em Evidências; Dor; Guia de Prática Clínica; Estudantes de Enfermagem. 


\section{Introducción}

\section{El dolor como quinto signo vital y su fundamentación}

El dolor es considerado el quinto signo vital y según la Asociación Internacional para el Estudio del Dolor (IASP) como "una experiencia sensorial y emocional no placentera que se asocia con daño tisular real o potencial, o que se describe desde el punto de vista de ese daño" (1), se encuentra dentro de las diez principales causas de consulta en los servicios de urgencias no solo en Colombia sino internacionalmente $(2,3)$.

El dolor como diagnóstico enfermero está considerado por la Asociación de Diagnósticos de Enfermería de Norteamérica (NANDA), tiene en cuenta la clasificación de dolor agudo y crónico y ha empleado la misma definición de la IASP complementando que el dolor es de inicio súbito o lento de cualquier intensidad de leve a grave con un final anticipado o previsible y de una duración inferior a 6 meses cuando se trata de un dolor agudo y una duración superior a 6 meses cuando se trata de dolor crónico. El dolor puede ser clasificado según su origen, duración, intensidad, localización y velocidad de conducción (4).

Según su origen: existen dos tipos de dolor, nociceptivo y neuropático, cada uno tiene una característica específica de presentación. Según su duración: puede encontrarse el dolor agudo y crónico. Según su intensidad: se clasifica en leve, moderado y severo; en el dolor leve interviene básicamente la piel por medio de los receptores de presión, temperatura, tacto fino, que pueden ser lesionados; es considerado leve si la escala visual numérica está entre 1 y 3 . En el dolor moderado está involucrada la epidermis y el tejido celular subcutáneo, se considera dolor moderado si la escala numérica está entre 4 y 6 . En el dolor severo se comprometen los músculos, las articulaciones, huesos y otros tejidos; se considera severo si la escala numérica está entre 7 y 10. Según su localización, puede ser localizado o difuso; el dolor difuso puede ser irradiado o referido. Según la velocidad de conducción puede ser clasificado como dolor rápido o lento (5).

Adicionalmente, es importante tener en cuenta que el dolor es un fenómeno complejo y multifactorial que depende de la interacción de factores fisiológicos, psicológicos y socioculturales; se han descrito diferencias en la percepción y manifestación relacionadas con condiciones étnicas o raciales (6).

El dolor no aliviado produce unos efectos psicosociales y psicológicos profundos en el paciente, lo cual puede influir en la recuperación de una enfermedad grave, modificar su funcionamiento físico y emocional, disminuir su calidad de vida y afectar su capacidad laboral.

Uno de los problemas comunes en el tratamiento del dolor es la oligoanalgesia, dentro de las causas de este problema se encuentran el no reconocimiento del dolor, fracaso para evaluar el dolor inicial, incumplimiento de las directrices de tratamiento del dolor, fracaso para documentar el dolor, evaluación de la adecuación del tratamiento y fracaso para satisfacer las expectativas del paciente con respecto al manejo del dolor. Así mismo, entre las causas del inadecuado manejo del dolor se señalan la deficiencia en la valoración, tratamiento farmacológico y documentación del dolor; además de la falta de información al paciente respecto al manejo del dolor (7).

Dada la importancia de la evaluación, manejo y control del dolor, éste se ha catalogado como el quinto signo vital, quedando al mismo nivel que la tensión arterial, pulso, frecuencia respiratoria y temperatura. A pesar de la importancia de este signo en Colombia son escasos los estudios acerca del dolor; la Asociación Colombiana para el estudio del dolor ha realizado esfuerzos en investigaciones por medio de entrevistas telefónicas, en su sexto estudio nacional del dolor del 2011 mostró que el 55.3\% de las personas presentaron dolor en la última semana y el $81.8 \%$ en el último año y el $27.5 \%$ de las personas consultaron en el servicio de urgencias a causa del dolor (8).

Las causas de infravaloración del dolor y el inadecuado tratamiento son multifactoriales, siendo los problemas más destacados la falta de formación y de información del profesional sanitario y el desconocimiento sobre la utilización de tratamientos de forma segura, efectiva y eficiente.

En un estudio multicéntrico, observacional y descriptivo realizado en 9 escuelas de enfermería de las universidades de la Comunidad Autónoma de Madrid (CAM) reportan la existencia de pobres conocimientos en dolor y malas actitudes ante dicho síntoma; proponen para mejorarlos, en el caso de los estudiantes, cambios en los programas curriculares de la diplomatura de enfermería, y en el caso de los profesionales, formación continuada, así como actualización de conocimientos en dicho tema (9).

La valoración integral del dolor incluye a personas incapaces de expresar su propio dolor como son: recién nacidos, adulto mayor con deterioro cognitivo, enfermos críticos, personas inconscientes, entre otros donde, es fundamental comprender cada una de estas incapacidades y utilizar herramientas validadas que permitan valorar adecuadamente el dolor y brindar intervenciones eficaces en planes individualizados (10).

\section{Necesidades del recurso humano e instituciones interesadas en orientar los procesos de formación}

Se ha identificado que el profesional de enfermería, en ocasiones, pasa por alto la valoración de este síntoma desagradable como es el dolor, lo cual puede deberse a desconocimiento, falta de tiempo, estrés laboral, situaciones que por ningún motivo deben excusar que el profesional 
brinde un cuidado con calidad, pero desafortunadamente la realidad demuestra que son múltiples las quejas en la satisfacción del usuario, en los diferentes servicios donde se reciben y atienden personas con necesidad del manejo del dolor, insatisfacción con el trato recibido por parte del personal de salud, especialmente relacionado con el trato digno y el respeto (11), como lo menciona Montealegre, el cuidado es la esencia de las competencias del profesional, entendiéndose como cuidado: prodigar, proveer, atender, administrar acciones tendientes a brindar comodidad, alivio, equilibrio biopsicosocial al individuo-familiacomunidad en los diferentes sistemas de atención en salud (12).

El dolor es un grave problema de salud pública en todo el mundo, es subvalorado y muchas veces no tratado adecuadamente por diversas razones de cultura, actitud, educación, política y logística. Teniendo en cuenta esto la legislación internacional en materia de derechos humanos de las Naciones Unidas lo declara en (1948) como un Derecho humano fundamental, así mismo existe el derecho a recibir el más alto nivel de salud, lo cual implica el alivio del dolor. Es importante destacar los avances de la OMS y la IASP para promover mejores prácticas analgésicas en todo el mundo, estableciendo fechas importantes como la "Semana Europea contra el Dolor", el "Día Mundial contra el Dolor" (13).

Estas y otras organizaciones a nivel mundial reconocen claramente que el alivio del dolor exige los más altos niveles profesionales y destacando esta importancia se ha incorporado la valoración del dolor como el quinto signo vital que debe ser valorado de forma continua en el paciente con herramientas validadas y adoptadas por los servicios de salud dentro de un protocolo institucional. Muchas instituciones de salud lo declaran como un derecho de los pacientes que, incluye el derecho a ser creído en las manifestaciones de dolor, a ser sometido a una evaluación y a un tratamiento adecuados del dolor, a que los resultados de esa evaluación se registren periódicamente, a recibir cuidados de profesionales sanitarios con formación y experiencia en la evaluación y el control del dolor, y a recibir unas estrategias eficaces para el alivio del dolor.

Es así como la valoración y el manejo del dolor ha pasado de ser una necesidad del paciente a ser un derecho que se exige y debe ser atendido con calidad para lograr el mayor beneficio posible en el paciente. La OMS describe que la calidad consiste en la ejecución de acuerdo a estándares de intervenciones de probada seguridad, que son económicamente accesibles a la población en cuestión, y que poseen la capacidad de producir un impacto positivo en la mortalidad, morbilidad, discapacidad y malnutrición.

La adopción de unas normas universales de tratamiento del dolor para los profesionales de la salud ha sido el empeño de varias asociaciones internacionales que han trabajado incansablemente para construir una cultura de la práctica basada en la evidencia y de esta forma, impulsar agentes de cambio que logren una gestión eficaz en la valoración y manejo del dolor.

La Joint Commission on Acreditation of Health Care Organitation (JCAHO) exige a las instituciones que coordinen actividades de garantía de calidad en un programa institucional integrado; necesariamente dentro de ese programa de garantía debe estar la atención en la valoración y manejo del dolor por el equipo interdisciplinario de salud (14).

Además, se considera fundamental que el enfermero desde su formación académica conozca la fisiología de los mecanismos del dolor, tipos y manifestaciones del mismo, los diferentes tratamientos farmacológicos y no farmacológicos, así como las implicaciones éticas, de seguridad del paciente, el enfoque teórico a través del cual puede brindar un cuidado de enfermería integral para el dolor agudo o crónico que presenta el ser humano en sus diferentes etapas vitales, y a través de la práctica de la investigación con aplicación de la Enfermería Basada en la Evidencia (EBE), generar nuevos conocimientos para mejorar la calidad del trabajo profesional ofrecido; sustentado en la rigurosidad del método científico y acorde a las necesidades del cuidado humanizado e individualizado que requiere el paciente (15).

De esta manera, el programa de enfermería de la Universidad Autónoma de Bucaramanga (UNAB) a través de su currículo tiene el propósito de "Formar integralmente profesionales de enfermería, con sólida fundamentación científica, social, humanística y fomentar en los estudiantes la actitud investigativa para detectar, planificar y desarrollar acciones en el campo de las áreas clínicas, de salud pública y administrativas que les permitan favorecer la creación de espacios interdisciplinarios de trabajo, reconocer la importancia del diálogo, trabajo en equipo y del enfoque holístico para el manejo de los problemas de salud", que sean profesionales con sólidos conocimientos y pensamiento crítico que le permita tomar decisiones con fundamento científico (16).

Es así como el programa de enfermería de la UNAB en el año 2014 estableció un convenio con la Asociación Profesional de Enfermeras de Ontario (RNAO) con el fin de obtener la certificación de buenas prácticas en enfermería (BPSO), certificado que representa la iniciativa de impactar en la atención de las personas a través de un desempeño institucional de calidad, cuyos resultados faciliten y fomenten buenas prácticas basadas en la evidencia científica al incorporar la implementación de tres Guías de Práctica Clínica (GPC) a nivel curricular, entre las cuales se encuentran: Valoración del riesgo y prevención de las úlceras por presión, prevención de caídas y lesiones derivadas de las caídas en personas mayores, y la de valoración y manejo del dolor. De esta última se hablará en este artículo. 
La determinación de incluir la guía de valoración y manejo del dolor dentro del programa de enfermería de la UNAB obedece a varios factores, entre los prioritarios identificar que el dolor es un tema crítico en la atención de las personas que lo padecen y el manejo adecuado es uno de los aspectos importantes y urgentes que se deben abordar en enfermería, por ello es trascendental la formación de los futuros profesionales de enfermería en el cuidado de la persona con dolor, por tanto, debe encontrarse inmerso en el currículo teniendo en cuenta las áreas tanto teóricas como prácticas.

El éxito en el manejo del dolor radica en la coordinación, el entrenamiento y el compromiso de todo el equipo de trabajo y de la educación que se brinde al paciente y sus cuidadores. Es así como, se toma como punto de partida fundamental para la implementación en el currículo del programa de enfermería de la UNAB, la guía de valoración y manejo del dolor de la RNAO, que tiene recomendaciones importantes para la formación del enfermero, entre ellas la recomendación 67: Todo programa que prepara a las enfermeras para la práctica clínica debe incluir contenido sobre el dolor basado en el currículo propuesto por la Internacional Association for the Study of Pain, (IASP), y examinar tanto la compresión teórica como la competencia clínica en el manejo del dolor (10).

Hay que resaltar en los contextos académicos las implicaciones éticas y legales que tiene el hecho de no utilizar las estrategias disponibles para la valoración y manejo del dolor, con el fin de que puedan incluirse planes integrales de cuidado que permitan cubrir necesidades de los pacientes con dolor y resolver los conflictos y los dilemas éticos en defensa de los pacientes y las familias (17).

Es pertinente reconocer que dentro de la organización RNAO se ha generado un movimiento internacional en el que se han involucrado instituciones de países como China, Japón, Australia, Sudáfrica, Chile, Colombia, Italia, Dubai y España entre otros, para trabajar aunadamente en la implantación de las guías de buenas prácticas, estos centros son evaluados tanto a lo largo del período de convenio con la RNAO como a su finalización del mismo. Los que obtienen la calificación positiva, reciben una acreditación como centro Best Practice Spotlight Organization (BPSO) por parte de RNAO (18). La certificación de centros BPSO, se hace a instituciones universitarias o sanitarias tales como hospitales, centros de larga estancia, centros de atención sanitaria y otros centros públicos de atención sanitaria que, mediante la implantación de dichas guías, permitirán la obtención de datos sobre el papel que juegan las enfermeras en la salud y el bienestar de los pacientes y determinar cómo la aplicación de las mencionadas guías ayudan a mejorar tanto los resultados clínicos de los pacientes como la eficacia y la eficiencia de los sistemas de salud participantes en los programas de implementación.

En Colombia actualmente hay dos instituciones de salud que están aplicando la guía de valoración y manejo del dolor de la RNAO, siendo reconocidas como instituciones BPSO, la Fundación Oftalmológica de Santander (FOSCAL) y la Fundación Cardioinfantil, en el ámbito académico, el programa de enfermería de la UNAB se ha vinculado como parte del proyecto internacional BPSO, en coherencia con el plan de desarrollo institucional UNAB, 2013-2018 y en cumplimiento de la perspectiva de procesos que permitan fortalecer la internacionalización como eje estratégico institucional.

A nivel de productividad y competitividad el establecimiento de alianzas estratégicas con la RNAO y las guías basadas en evidencia, pretende mejorar el nivel de los profesionales en formación al asegurar brindar un cuidado con altos estándares de calidad dentro del marco de seguridad del paciente. En el plan de desarrollo de la UNAB dentro del escenario de apuesta al 2,018 destaca que: "La comunidad UNAB piensa global y actúa localmente, articulando la academia a las necesidades de la región y a los contextos nacional e internacional en donde la investigación pertinente, articulada al desarrollo académico y a las expectativas sociales, contribuye al mejoramiento de la oferta curricular y a la solución de la problemática del entorno productivo y social".

\section{Enfermería basada en la evidencia y guías de buenas prácticas clínicas en los procesos de formación}

La enfermería basada en la evidencia es un concepto que para la disciplina ha evolucionado históricamente, teniendo en cuenta que en sus orígenes se limitaba exclusivamente a demostrar una serie de habilidades para cuidar la vida y preservar la especie (19) considerada en la edad media una labor inferior realizada por mujeres que sentían compasión por el ser humano.

Posteriormente, con el avance de las ciencias médicas se hizo necesario entrenar a estas mujeres, pero eran los médicos quienes transmitían el conocimiento, lo que generaba un cuidado dependiente por completo de las indicaciones médicas; sin embargo, es alrededor del siglo XIX, en 1854, cuando Florence Nightingale a través de su quehacer enfermero y utilizando sus conocimientos en matemáticas y los métodos estadísticos aprendidos, ilustrados en el diagrama del área polar, logró mejorar las condiciones de vida de los soldados heridos en la guerra de Crimea, entregando a la historia de la disciplina las primeras evidencias de un cuidado que impactó en el control y reducción de la mortalidad secundaria a infecciones de un $40 \%$ al $2 \%$. Posteriormente, esta información fue usada como herramienta para mejorar el cuidado sanitario en los hospitales militares (20).

No obstante, es preciso resaltar la gran brecha que existe en la evolución de la EBE, puesto que es ampliamente conocido que esta estrategia metodológica aparece en el siglo XX en los años ochenta, en la Escuela de Medicina de la Universidad de McMaster de Ontario como un modelo denominado medicina basada en la evidencia (MBE), donde 
un grupo de clínicos y epidemiólogos reunidos crearon dicho movimiento, liderado por Sackett, sustentando que las decisiones de la práctica médica no estaban fundamentadas en resultados provenientes de la investigación(21).

Sin embargo, solo hasta finales del siglo XX, específicamente en 1997, tiene su origen la enfermería basada en la evidencia (EBE) en los ámbitos académicos de la Universidad de York en el Reino Unido, donde surge como una necesidad de unir la investigación y las prácticas, las enfermeras crean la necesidad de utilizar el conocimiento para la acción y desarrollar una herramienta de alta calidad que se reconociera como un elemento clave en el cuidado asistencial, para de esta manera lograr un impacto positivo en los indicadores de calidad (22).

Concibiéndose así la EBE como "Proceso por el cual las enfermeras toman decisiones clínicas, usando las mejores pruebas disponibles sustentadas en la investigación, su experiencia clínica y las preferencias del paciente, en el contexto de los recursos disponibles" (23).

Hoy en día las instituciones de salud se preocupan fundamentalmente por la actualización tecnológica para ser competitivas, pero preocupa poco, el hecho de que sin recurso humano de todo tipo, entre otros, enfermeros profesionales, de nada servirá la tecnología para alcanzar niveles satisfactorios de calidad en la atención. Se requiere entonces de unos profesionales idóneos con capacidad crítica que puedan tomar decisiones propias de su rol profesional, con acciones independientes que garanticen la seguridad de los usuarios, por ello desde la formación del estudiante es necesario propender en la importancia de la evidencia científica para la toma de decisiones.

Las guías de cuidado basadas en evidencia científica permiten disminuir las dificultades de los enfermeros en su práctica diaria, facilitando intervenciones autónomas con base en resultados de tal manera que sea posible ejercer un rol específico e independiente en el equipo multidisciplinario donde, en ambientes reales de trabajo profesional, se materialice la definición de enfermería dada por la Ley 266 de 1996, "Enfermería es una profesión liberal y una disciplina de carácter social.", buscando siempre el establecimiento y mantenimiento de una relación de ayuda al paciente, viendo más allá de unos cuidados físicos y recordando que el ser humano constituye una unidad biológica, psicológica, social, espiritual y cultural (24).

Este cuidado basado en evidencia científica se logra a través de una sólida formación académica y humana en pro del desarrollo de su autonomía personal, profesional, con motivación hacia la capacidad de autoformación para asumir así, los diferentes roles que debe cumplir en un sistema de salud cambiante, en donde su liderazgo y oportuno juicio clínico lleve al profesional a brindar a los pacientes cuidados humanos eficaces, con calidad y excelencia profesional.
Todo esto conduce a preguntas sobre: ¿Qué se está haciendo para lograr esta meta en el Programa de Enfermería UNAB?, ¿Con qué bases conceptuales y científicas se está trabajando?, se encuentra que las respuestas a estas preguntas están inmersas en la evidencia científica que proporcionan las guías de prácticas clínicas y demostradas en las guías clínicas de la RNAO.

Lo anterior aporta de forma significativa no solo al programa, docentes y estudiantes sino a la disciplina y profesión de enfermería los conocimientos esenciales para la práctica, la investigación y el desarrollo de la evidencia científica(25).

De esta manera, el programa enfermería busca innovar con un currículo globalizado que responda a las necesidades tanto del país como del mundo, permitiendo a los estudiantes acceder a estudios de postgrado, a becas y pasantías en otros países y a la posibilidad de ejercer la profesión a nivel nacional e internacional. Es por ello que también durante la carrera se hace énfasis en la enseñanza del inglés como segunda lengua y fomentar la investigación en los estudiantes en cada uno de los cursos del plan de estudios.

Como estrategia de enseñanza y aprendizaje y como resultado de este, apropiarse de la enfermería basada en la evidencia es una herramienta primordial para conocer y comprender las necesidades de los individuos, las familias y las comunidades con relación a la experiencia de la salud, y en beneficio del bienestar de las personas.

De esta manera, este artículo reflexivo busca presentar la experiencia de la primera fase de implementación en el currículo del programa de enfermería de la guía de buenas prácticas clínicas para la valoración y manejo del dolor de la RNAO.

\section{Discusión}

La metodología utilizada para la implementación de la guía de valoración y manejo del dolor de la RNAO tuvo como marco de referencia el Proyecto Educativo Institucional, el Proyecto educativo del programa de la UNAB, la herramienta de Implementación de buenas prácticas propuesta por la RNAO y el levantamiento de información que permitiera identificar los vacíos y las acciones de implementación en los contenidos curriculares respecto al uso de recomendaciones de práctica clínica para la valoración y manejo del dolor en el currículo del programa de enfermería, a continuación se desarrollan estos apartados

\section{Marco institucional y conocimiento de la guía de práctica clínica de valoración y manejo del dolor la RNAO en la UNAB}

El conocimiento del trabajo desarrollado por la RNAO se inició en agosto de 2013 en el programa de enfermería 
UNAB, durante la participación en el primer congreso internacional de enfermería basada en la evidencia organizado por la FOSCAL, cuyo eje temático fue la enfermería basada en la evidencia y las guías clínicas de buenas prácticas de enfermería de la RNAO, teniendo en cuenta que el objetivo de ésta es lograr transformar la cultura de la enfermería y asegurarse de que su práctica clínica esté completamente basada en la evidencia como eje fundamental para mejorar la atención de los pacientes y sus resultados de salud.

La UNAB, como institución educativa de alta calidad, expresa la importancia de participar y proponer un trabajo a futuro con las guías clínicas de la RNAO e implementarlas en el plan de estudios del programa de enfermería. Para fortalecer este trabajo se contó con la alianza estratégica de la FOSCAL, principal sitio de práctica formativa que tienen los estudiantes del programa de enfermería desde el tercer semestre; esta oportunidad facilitaría el empoderamiento por parte de los estudiantes de las guías de buenas prácticas clínicas RNAO en el cuidado asistencial, bajo el acompañamiento de los docentes de la FOSCAL vinculados a la UNAB, quienes tienen el conocimiento y han sido actores fundamentales a nivel institucional en la implementación de las guías de buenas prácticas clínicas.

El propósito del convenio con la RNAO apoya el cumplimiento de la misión del programa de enfermería UNAB, "Somos un programa de enfermería con proyección internacional orientado a la formación integral de enfermeros con alta calidad humana, ética y científica, con capacidad de liderazgo para dar cuidado integral de salud a la persona, familia y comunidad en todas las etapas del proceso salud enfermedad y generar proyectos en salud que diversifiquen las formas de ejercicio profesional de la enfermería"

Asimismo apoya la visión UNAB: "En el 2018 el programa de enfermería tendrá acreditación nacional y será líder en la formación de profesionales de Enfermería, capaces de promover el desarrollo de la disciplina en beneficio de la profesión, las instituciones y la sociedad nacional e internacionalmente, gracias a la calidad innovadora de su pregrado y postgrados, la incorporación de las TIC, a la formación continua de sus docentes y al impacto ético, social, ambiental y científico de sus egresados que aportarán soluciones para garantizar el mejoramiento constante de la calidad de vida de las personas y el bienestar integral de la sociedad".

Es así como, el Programa de Enfermería viene trabajando en la actualización del plan de estudios que favorece el fortalecimiento del rol investigativo, la apropiación de estrategias como la evidencia científica y la implementación de las guías de prácticas clínicas.

En coherencia con el trabajo práctico que los estudiantes realizan en la FOSCAL se decidió trabajar con las mismas guías de buenas prácticas clínicas de la RNAO en la parte académica: Valoración del riesgo y prevención de las úlceras por presión, prevención de caídas y lesiones derivadas de las caídas en personas mayores, y la de valoración y manejo del dolor, sin obviar la posibilidad de trabajar con otras guías que están siendo evaluadas actualmente por los grupos de docentes a cargo de los cursos de cuidado de las comunidades y salud mental, de igual manera se plantearon los objetivos de implementación (Tabla 1).

\section{Metodología de Implementación a nivel organizacional: Best Practice Spotlight Organization ( $\mathrm{BPSO}^{\circledR}$ )}

Capacitación: Para cumplir con los anteriores objetivos, durante el segundo semestre del año 2015, se desarrolló un programa de capacitación de 40 horas durante cinco días,

Tabla 1. Objetivos de la implementación de la guía clínica de valoración y manejo del dolor de la RNAO.

\begin{tabular}{cl}
\hline $\mathbf{1}$ & Transversalizar en el currículo de pregrado las guías clínicas de la RNAO. \\
\hline $\mathbf{2}$ & $\begin{array}{l}\text { Dar a conocer a docentes y estudiantes la importancia de brindar cuidado de enfermería } \\
\text { basado en evidencia científica. }\end{array}$ \\
\hline $\mathbf{3}$ & $\begin{array}{l}\text { Dar seguridad al usuario durante el cuidado de enfermería dado pordocentes y estudiantes } \\
\text { en las prácticas formativas. }\end{array}$ \\
\hline $\mathbf{4}$ & Internacionalizar el currículo permitiendo la movilidad docente y estudiantil. \\
\hline $\mathbf{5}$ & $\begin{array}{l}\text { Desarrollar investigaciones multicéntricas que mejoren los procesos de cuidado basados } \\
\text { en evidencia científica. }\end{array}$ \\
\hline $\mathbf{6}$ & $\begin{array}{l}\text { Formar estudiantes que brinden cuidado de enfermería con capacidad crítica y ética. } \\
\text { práctica formativa. }\end{array}$ \\
\hline $\mathbf{8}$ & $\begin{array}{l}\text { Ser participe en la difusión del conocimiento apoyado en los resultados de la } \\
\text { implementación de las guías a través de indicadores de resultado tanto en la academia } \\
\end{array}$ \\
\hline
\end{tabular}

Fuente: Convenio RNAO-Programa de enfermería 2015 
dirigido a los docentes de planta del programa de enfermería de la UNAB, facilitado por representantes de la Asociación de Enfermeras de Ontario, denominada metodología de implementación a nivel organizacional: BPSO, que tuvo como objetivo compartir desde la perspectiva de la experiencia de la asociación, las principales recomendaciones a tenerse en cuenta para la implementación de las guías de buenas Prácticas Clínicas (GBPs), también se dio a conocer el modelo de entrenamiento para los docentes con asignación de hora cátedra como: Best Practice Champions, utilizando los recursos de la RNAO, lo anterior, como un enfoque para la creación de culturas de práctica basada en la evidencia.

La capacitación al grupo de docentes del programa de enfermería de la UNAB ofreció conocimientos en los fundamentos históricos de la práctica basada en la evidencia, en la creación de una cultura basada en la evidencia en el cuidado de la salud, en el uso de la evidencia en la práctica y la academia, en estrategias de transferencia de conocimiento en el contexto de la investigación, la educación y la práctica, además de la importancia de la colaboración entre la academia y las instituciones de servicio.

Durante la misma se desarrollaron aspectos muy importantes como:

- Identificar los factores que facilitan o pueden ser barreras para la sostenibilidad.

- Comprender la importancia de la planificación para el sostenimiento de las mejores prácticas
- Introducir enfoques, modelos, herramientas o recursos conocidos para ayudar con la planificación para mantener las mejores prácticas.

- Identificar cambios en la infraestructura clave que ayudarán en la incorporación de las guías en el sistema para apoyar la sostenibilidad.

- Evaluar la efectividad de las diversas prácticas de sostenibilidad en la creación de una cultura basada en la evidencia.

- Conocer el sistema de indicadores de calidad de enfermería para informes y evaluación (NQuIRE).

- Estrategias prometedoras para la práctica basada en la evidencia Sostenible.

Posterior a este entrenamiento de BPSO $\AA$, el programa de enfermería UNAB desarrolló una agenda de trabajo durante las reuniones del comité docente, donde se organizaron tres grupos para asumir las responsabilidades de la implementación de cada una las tres guías de buenas prácticas clínicas de la RNAO, entre ellas y como parte de éste artículo de reflexión, la de valoración y manejo del dolor en el currículo de enfermería, para tal fin se asignó un docente coordinador por grupo que liderara el proceso de implementación en el plan de estudios en forma transversal.

Teniendo en cuenta la herramienta de implementación de buenas prácticas, cada uno de los equipos de trabajo presentaron el esquema de identificación del problema, revisión y selección del conocimiento (Figura 1).

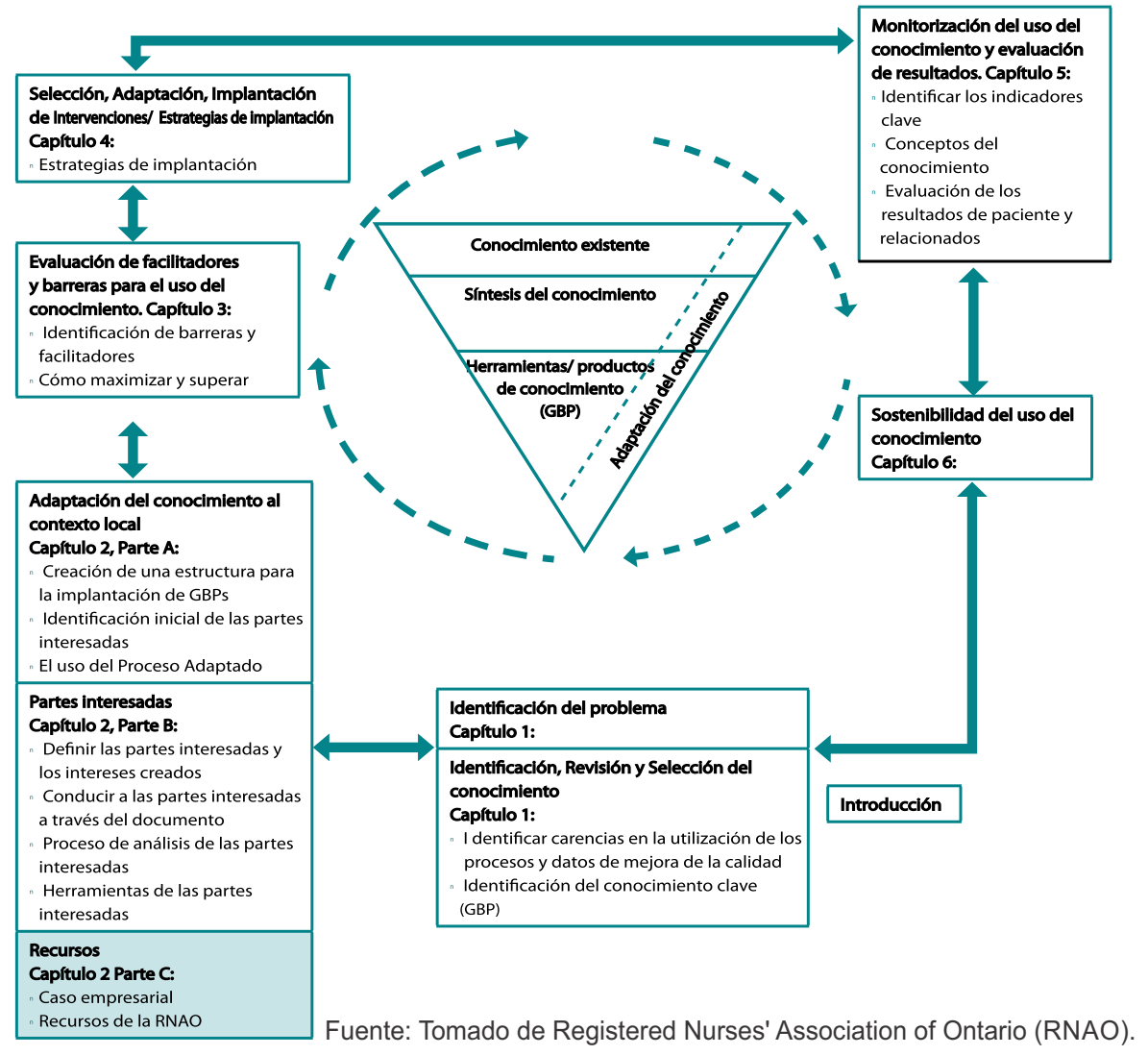

Figura 1. Guía de buenas prácticas en enfermería. Herramientas de implantación de buenas prácticas. Knowledge-to-Action Process, año 2012. 
Se realizó un ejercicio inicial de reconocer los facilitadores y barreras en este proceso de implementación de la guía propuesta en las herramientas de implantación de buenas prácticas (26).

\section{Adaptación de la guía de valoración y manejo del dolor en el contexto académico}

En este proceso de adaptación se establecieron 3 fases:

\section{Fase 1: Puesta en marcha}

En esta fase se contemplaron actividades relacionadas con la preparación para el inicio de un proceso de adaptación. Esto implicó el establecimiento del comité institucional o equipo de implantación, en el comité docente del programa de enfermería de la UNAB el cual se conformó con tres docentes de tiempo completo, y una docente de medio tiempo.

En la guía de dolor se seleccionaron los temas de valoración, manejo del dolor que incluya estrategias farmacológicas y no farmacológicas. Un área importante a considerar fue la disponibilidad de las guías de práctica clínica relevantes con niveles de evidencia (Tabla 2), lo que permitirá identificar los recursos a utilizar en la plataforma moodle, denominada en la UNAB, Tecnología Aplicada a la Enseñanza para el Mejoramiento del Aprendizaje (TEMA), la comunicación a través de las redes virtuales, Skype, además de las habilidades y el conocimiento de los docentes sobre valoración y manejo del dolor y las investigaciones en este campo.

Posteriormente se estableció un plan de trabajo para el comité del grupo, y se asignaron responsabilidades, se establecieron los recursos disponibles, fechas de entrega y con un cronograma de actividades que incluía reuniones semanales.

Para la puesta en marcha de este plan de trabajo se tuvo en cuenta la cartilla: Herramientas de implementación de buenas prácticas, donde a la fecha nos encontramos en el capítulo de Estrategias de implementación (26).

\section{Fase 2: Adaptación}

En esta fase se establecieron por parte del grupo el alcance y la finalidad, a través de la metodología: PIPRE, que contempla:

- Población destinataria: docentes cátedra, docentes de otras disciplinas, estudiantes de Enfermería de I-VIII semestre.

- Intervención de interés: valoración, estrategias farmacológicas y no farmacológicas.

- Profesionales a los que se dirige la implementación: estudiantes del programa de enfermería de I-VIII semestre.

- Resultados deseados: Intervenciones y cuidados de enfermería con guías basadas en evidencia.

- Entorno o contexto de salud donde se implantará la guía: contexto académico, sitios de prácticas formativas.

En coherencia con los cursos de formación del currículo del programa de enfermería y buscando tener continuidad del contexto académico con las prácticas formativas que realizan los estudiantes, se escogió la guía de valoración y manejo del dolor, tercera edición, ya que corresponde a una guía que actualmente está implementada en el área asistencial de la institución FOSCAL, con el fin de garantizar continuidad y correlación de los contenidos teóricos aprendidos en el aula de clase con el contexto real de las prácticas institucionales.

En consenso el comité de la guía de valoración y manejo de dolor tomó la decisión de seleccionar las veinte recomendaciones de la guía basadas en evidencia de revisión sistemática que ofrece para las enfermeras y otros miembros del equipo interprofesional, recomendaciones para la práctica en las etapas de valoración, planificación, implantación, valoración; para el contexto académico incluye recomendaciones para la formación y a nivel administrativo contempla recomendaciones para la organización y directrices (Tabla 3 ).

Tabla 2. Adaptación propuesta por la RNAO de los niveles de evidencia e interpretación de los tipos de estudio.

\begin{tabular}{cl}
\hline la & $\begin{array}{l}\text { Evidencia obtenida del metaanálisis o de la revisión sistemática de ensayos controlados } \\
\text { aleatorios. }\end{array}$ \\
\hline Ib & Evidencia obtenida de al menos un estudio controlado aleatorio. \\
\hline Ila & Evidencia obtenida de al menos un estudio bien diseñado controlado no aleatorio. \\
\hline Ilb & $\begin{array}{l}\text { Evidencia obtenida de al menos una muestra de otro tipo de estudio bien diseñado, } \\
\text { cuasiexperimental, no aleatorio. }\end{array}$ \\
\hline III & $\begin{array}{l}\text { Evidencia obtenida de estudios descriptivos bien diseñados, no experimentales, como } \\
\text { son los estudios comparativos, estudios de correlación y estudios de caso. }\end{array}$ \\
\hline IV & $\begin{array}{l}\text { Evidencia obtenida de la opinión de los informes elaborados por un comité de expertos } \\
\text { y/o las experiencias de autoridades. }\end{array}$ \\
\hline
\end{tabular}

Fuente: Registered Nurses' Association of Ontario. Assessment and Management of Pain (3rd ed.). Toronto, Canada: Registered Nurses' Association of Ontario.2013. 
Tabla 3. Cuadro de recomendaciones basadas en la evidencia para la guía de valoración y manejo del dolor propuesta por la RNAO.

\section{Recomendaciones para la practica}

Nivel de

evidencia

$1.0 \quad 1.1$ Detectar la presencia, o riesgo, de cualquier tipo de dolor: al ingreso o en consulta con el profesional responsable, después de

Valoración un cambio en la situación clínica y antes, durante y después de un procedimiento.

1.2 Realizar una valoración integral del dolor en las personas a las Ib que se les haya detectado la presencia de dolor, o el riesgo de cualquier tipo de dolor utilizando un enfoque sistemático y herramientas validadas apropiadas.

1.3 Realizar una valoración integral del dolor en personas incapaces III de expresar su propio dolor, utilizando una herramienta validada.

1.4 Explorar las creencias, el conocimiento y el nivel de compresión III sobre el dolor y el manejo del dolor de la persona.

\begin{tabular}{clc}
\cline { 2 - 3 } & 1.5 Registrar las características del dolor del paciente. & Ila \\
\hline Planificación & $\begin{array}{l}\text { 2.1 Colaborar con la persona a identificar sus metas para el manejo } \\
\text { del dolor y las estrategias adecuadas para garantizar un enfoque } \\
\text { integral para el plan de cuidados. }\end{array}$ & lb
\end{tabular}

2.2 Establecer un plan integral de cuidados que incorpore los III objetivos de la persona y del equipo interprofesional y que contemple: Evaluación de los resultados; las creencias, el conocimiento y el nivel de compresión de la persona y las características personales y las características del dolor.

\begin{tabular}{|c|c|c|}
\hline \multirow[t]{3}{*}{$\begin{array}{c}3.0 \\
\text { Implantación }\end{array}$} & $\begin{array}{l}\text { 3.1 Implantar el plan de manejo del dolor utilizando principios que } \\
\text { maximicen la eficacia y minimicen los efectos adversos de las } \\
\text { intervenciones farmacológicas incluyendo: un enfoque deanalgesia } \\
\text { multimodal, un cambio de opioides, la prevención, la valoración y el } \\
\text { manejo de los efectos adversos, prevención, evaluación y manejo } \\
\text { de los riesgos de los opioides. }\end{array}$ & Ib \\
\hline & $\begin{array}{l}\text { 3.2 Evaluar las intervenciones no farmacológicas (físicas y } \\
\text { psicológicas) para la eficacia de las intervenciones farmacológicas. }\end{array}$ & $\mathrm{Ib}$ \\
\hline & $\begin{array}{l}\text { 3.3 Formar a la persona, su familia y los cuidadores sobre las } \\
\text { estrategias de manejo del dolor en su plan de cuidados y abordar } \\
\text { las preocupaciones y creencias erróneas. }\end{array}$ & Ib \\
\hline \multirow[t]{2}{*}{$\begin{array}{l}4.0 \\
\text { Valoración }\end{array}$} & $\begin{array}{l}\text { 4.1 Volver a valorar la respuesta de la persona a las intervenciones } \\
\text { de manejo de dolor constantemente utilizando la misma herramienta } \\
\text { de valoración. La frecuencia de las valoraciones vendrá } \\
\text { determinada por: la presencia del dolor, la intensidad del dolor, la } \\
\text { estabilidad de la situación clínica del paciente, el tipo de dolor, la } \\
\text { institución de salud. }\end{array}$ & $\mathrm{Ilb}$ \\
\hline & $\begin{array}{l}4.2 \text { Comunicar y registrar las respuestas de la persona al plan del } \\
\text { manejo del dolor. }\end{array}$ & $\mathrm{Ib}$ \\
\hline
\end{tabular}




\begin{tabular}{|c|c|c|}
\hline \multirow[t]{4}{*}{$\begin{array}{l}5.0 \\
\text { Educación }\end{array}$} & $\begin{array}{l}\text { 5.1 Las instituciones educativas deben incorporar esta guía, en los } \\
\text { programas curriculares interprofesionales y de enfermería para los } \\
\text { programas de enfermería, formación profesional y medicina para } \\
\text { promover la práctica basada en la evidencia. }\end{array}$ & $\mathrm{Ilb}$ \\
\hline & $\begin{array}{l}5.2 \text { Incorporar los contenidos sobre las estrategias de transferencia } \\
\text { del conocimiento en los programas de formación para los } \\
\text { profesionales de la salud para trasladar a la práctica la evidencia } \\
\text { relacionada con el manejo del dolor. }\end{array}$ & $\mathrm{Ilb}$ \\
\hline & $\begin{array}{l}\text { 5.3 Promover la for mación y la colaboración interporfesional en } \\
\text { relación con la valoración y manejo del dolor en las instituciones } \\
\text { académicas. }\end{array}$ & Ib \\
\hline & $\begin{array}{l}\text { 5.4 Los profesionales de salud deben participar en la formación } \\
\text { continua para mejorar los conocimientos específicos y las } \\
\text { habilidades relacionadas con la valoración y manejo del dolor de } \\
\text { forma efectiva basándose en la guía de valoración y manejo del } \\
\text { dolor ( } 3 \text { ed). }\end{array}$ & IV \\
\hline \multirow{4}{*}{$\begin{array}{l}\quad 6.0 \\
\text { Organizacione } \\
\text { s y directrices }\end{array}$} & $\begin{array}{l}\text { 6.1 Establecer la valoración y manejo del dolor como una prioridad } \\
\text { estratégica en salud. }\end{array}$ & IV \\
\hline & $\begin{array}{l}\text { 6.2 Establecer un modelo de cuidados para apoyar la colaboración } \\
\text { interprofesional para la valoración y manejo del dolor eficaz. }\end{array}$ & $\mathrm{Ilb}$ \\
\hline & $\begin{array}{l}\text { 6.3Utilizar el proceso de transferencia del conocimiento y las } \\
\text { estrategias multimodales en las instituciones para ayudar a los } \\
\text { profesionales de la salud a utilizar en la práctica la mejor evidencia } \\
\text { en la valoración y manejo del dolor. }\end{array}$ & III \\
\hline & $\begin{array}{l}6.4 \text { Utilizar un enfoque sistemático para toda la institución para } \\
\text { implantar la guía de buenas prácticas Valoración y manejo del dolor } \\
\text { ( } 3 \text { ed) y proporcionar los recursos y los apoyos administrativos e } \\
\text { institucionales para facilitar su adopción. }\end{array}$ & IV \\
\hline
\end{tabular}

Fuente: Registered Nurses' Association of Ontario. Assessment and Management of Pain (3rd ed.). Toronto, Canada: Registered Nurses'Association of Ontario.2013.

\section{Fase 3: Partes interesadas}

Se identificaron las partes interesadas, que para el caso de la guía de buenas prácticas clínicas en valoración y manejo del dolor serían a nivel de individuos: estudiantes, docentes de planta, docentes de cátedra, docentes de ciencias básicas; como grupo el programa de enfermería y como institución: El departamento de enfermería de la FOSCAL, FOSCAL Internacional junto con las directivas de la UNAB.

Una vez identificadas las partes interesadas se realizó el análisis de la brecha entre la GBPs para la valoración y manejo del dolor y las guías cátedra del plan de estudios del programa, para lo cual se diseñó una matriz donde se reportaron las recomendaciones para la práctica en lo concerniente a valoración, planificación, implementación, educación y organización y directrices; realizando el comparativo con lo declarado en las guías cátedra. En el caso de la guía de valoración y manejo del dolor, al hacer la revisión de los contenidos declarados en las guías cátedra versus recomendaciones propuestas en las guía, se identificaron los siguientes resultados (Tabla 4) teniendo en cuenta la malla curricular del programa que está organizada por los componentes básico, específico y de profundización. En la Figura 2 se evidencia la malla curricular con los componentes y cada uno de los cursos que hacen parte del plan de estudios.

Respecto a la recomendación de valoración en el componente básico no se encontraron declaradas temáticas relacionadas con este aspecto. En relación con el componente específico I se identifica que los cursos de cuidado a las comunidades II, III y cuidado en unidades especiales incluyen temáticas en relación a la valoración, en especial el curso de unidades especiales, haciendo uso de las diferentes escalas.

En relación al curso de cuidado a las comunidades, se trabaja el dolor desde la perspectiva del sujeto en situación de cronicidad pero no son declaradas escalas de valoración. 
Tabla 4. Levantamiento de contenidos declarados en las guías cátedra versus recomendaciones propuestas en la guía de valoración y manejo del dolor RNAO.

\begin{tabular}{|c|c|c|c|c|}
\hline \multicolumn{2}{|c|}{ Recomendación según guía RNAO(III edición-2013) } & \multirow[t]{2}{*}{$\begin{array}{l}\text { Componente } \\
\text { básico }\end{array}$} & \multirow{2}{*}{$\begin{array}{c}\text { Componente } \\
\text { Específico I } \\
\text { Cuidado } \\
\text { paliativo y } \\
\text { manejo del } \\
\text { dolor } \\
\text { apoyo de } \\
\text { cuidadores. }\end{array}$} & \multirow{2}{*}{$\begin{array}{c}\text { Componente } \\
\text { Específico II } \\
\text { En educación } \\
\text { para la salud a } \\
\text { personas } \\
\text { mayores se } \\
\text { trabaja sobre } \\
\text { dolor articular, } \\
\text { signos y } \\
\text { síntomas como } \\
\text { dolor de cabeza }\end{array}$} \\
\hline \multirow{5}{*}{$\begin{array}{c}1.0 \\
\text { Valoración }\end{array}$} & $\begin{array}{l}\text { 1.1 Detectar la presencia, o riesgo, de } \\
\text { cualquier tipo de dolor: al ingreso o en } \\
\text { consulta con el profesional responsable, } \\
\text { después de un cambio en la situación clínica } \\
\text { y antes, durante y después de un } \\
\text { procedimiento. Nivel de evidencia lb }\end{array}$ & & & \\
\hline & $\begin{array}{l}\text { 1.2 Realizar una valoración integral del dolor } \\
\text { en las personas a las que se les haya } \\
\text { detectado la presencia de dolor, o el riesgo de } \\
\text { cualquier tipo de dolor utilizando un enfoque } \\
\text { sistemático y herramientas validadas } \\
\text { apropiadas. Nivel de evidencia lb }\end{array}$ & & $\begin{array}{c}\text { Cuidado } \\
\text { paliativo y } \\
\text { manejo del } \\
\text { dolor } \\
\text { Valoración del } \\
\text { dolor torácico. } \\
\text { Valoración del } \\
\text { paciente con } \\
\text { dolor abdominal }\end{array}$ & $\begin{array}{c}\text { Se registra si } \\
\text { presenta o no } \\
\text { dolor en el caso } \\
\text { de los programas }\end{array}$ \\
\hline & $\begin{array}{c}\text { 1.3 Realizar una valoración integral del dolor } \\
\text { en personas incapaces de expresar su propio } \\
\text { dolor, utilizando una herramienta validada. } \\
\text { Nivel de evidencia III }\end{array}$ & & $\begin{array}{c}\text { Valoración e } \\
\text { interpretación } \\
\text { de las escalas } \\
\text { de dolor- } \\
\text { Cuidado } \\
\text { Holístico de } \\
\text { Enfermería II: } \\
\text { Escalas de } \\
\text { dolor }\end{array}$ & \\
\hline & $\begin{array}{c}\text { 1.4 Explorar las creencias, el conocimiento y } \\
\text { el nivel de compresión sobre el dolor y el } \\
\text { manejo del dolor de la persona. Nivel de } \\
\text { evidencia III }\end{array}$ & & & $\begin{array}{c}\text { Creencias } \\
\text { sobre el manejo } \\
\text { del dolor }\end{array}$ \\
\hline & $\begin{array}{l}\text { 1.5 Registrar las características del dolor del } \\
\text { paciente. Nivel de evidencia lla }\end{array}$ & & & $\begin{array}{c}\text { Registro de } \\
\text { características } \\
\text { como localización, } \\
\text { intensidad de } 1 \text { a } 10\end{array}$ \\
\hline \multirow[b]{2}{*}{ Planificación } & $\begin{array}{l}\text { 2.1 Colaborar con la persona a identificar sus } \\
\text { metas para el manejo del dolor y las } \\
\text { estrategias adecuadas para garantizar un } \\
\text { enfoque integral para el plan de cuidados. } \\
\text { Recomendación lb }\end{array}$ & & & \\
\hline & $\begin{array}{l}2.2 \text { Establecer un plan integral de cuidados } \\
\text { que incorpore los objetivos de la persona y } \\
\text { del equipo interprofesional y que contemple: } \\
\text { evaluación de los resultados; las creencias, el } \\
\text { conocimiento y el nivel de compresión de la } \\
\text { persona y las características personales y las } \\
\text { características del dolor. Nivel de evidencia III }\end{array}$ & & & \\
\hline
\end{tabular}




\begin{tabular}{|c|c|c|c|}
\hline \multirow{3}{*}{$\begin{array}{c}3.0 \\
\text { Implantación }\end{array}$} & $\begin{array}{l}3.1 \text { Implantar el plan de manejo del dolor } \\
\text { utilizando principios que maximicen la eficacia } \\
\text { y minimicen los efectos adversos de las } \\
\text { intervenciones farmacológicas incluyendo: un } \\
\text { enfoque de analgesia multimodal, un cambio } \\
\text { de opioides, la prevención, la valoración y el } \\
\text { manejo de los efectos adversos, prevención, } \\
\text { evaluación y manejo de los riesgos de los } \\
\text { opioides. Nivel de evidencia lb }\end{array}$ & $\begin{array}{l}\text { Farmacocinética } \\
\text { y farmacodina } \\
\text { mia de los } \\
\text { opioides }\end{array}$ & $\begin{array}{l}\text { Control del } \\
\text { Dolor en el } \\
\text { Trabajo de } \\
\text { Parto: Técnicas } \\
\text { Farmacológicas } \\
\text { y no } \\
\text { Farmacológicas } \\
\text {. }\end{array}$ \\
\hline & $\begin{array}{c}\text { 3.2 Evaluar las intervenciones no } \\
\text { farmacológicas (físicas y psicológicas) para la } \\
\text { eficacia de las intervenciones farmacológicas. } \\
\text { Nivel de evidencia lb }\end{array}$ & & \\
\hline & $\begin{array}{c}\text { 3.3 Formar a la persona, su familia y los } \\
\text { cuidadores sobre las estrategias de manejo } \\
\text { del dolor en su plan de cuidados y abordar las } \\
\text { preocupaciones y creencias erróneas. Nivel } \\
\text { de evidencia lb }\end{array}$ & & $\begin{array}{l}\text { Abordaje al } \\
\text { cuidador sobre } \\
\text { el manejo del } \\
\text { dolor. }\end{array}$ \\
\hline \multirow[t]{2}{*}{$\begin{array}{l}4.0 \text { Valoración } \\
\text { (Revaloración) }\end{array}$} & $\begin{array}{c}\text { 4.1 Volver a valorar la respuesta de la } \\
\text { persona a las intervenciones de manejo de } \\
\text { dolor constantemente utilizando la misma } \\
\text { herramienta de valoración. La frecuencia de } \\
\text { las valoraciones vendrá determinada por: la } \\
\text { presencia del dolor, la intensidad del dolor, la } \\
\text { estabilidad de la situación clínica del paciente, } \\
\text { el tipo de dolor, la institución de salud. Nivel } \\
\text { de evidencia llb }\end{array}$ & & \\
\hline & $\begin{array}{c}\text { 4.2 Comunicar y registrar las respuestas de la } \\
\text { persona al plan del manejo del dolor. } \\
\text { Recomendación lb }\end{array}$ & & \\
\hline \multirow{3}{*}{ Educación } & $\begin{array}{c}5.1 \text { Las instituciones educativas deben } \\
\text { incorporar esta guía, en los programas } \\
\text { curriculares interprofesionales y de } \\
\text { enfermería para los programas de enfermería, } \\
\text { formación profesional y medicina para } \\
\text { promover la práctica basada en la evidencia. } \\
\text { Nivel de evidencia llb }\end{array}$ & & \\
\hline & $\begin{array}{l}5.2 \text { Incorporar los contenidos sobre las } \\
\text { estrategias de transferencia del conocimiento } \\
\text { en los programas de formación para los } \\
\text { profesionales de la salud para trasladar a la } \\
\text { profesionales de la salud para trasladar } \\
\text { práctica la evidencia relacionada con el } \\
\text { manejo del dolor. Nivel de evidencia Ilb }\end{array}$ & & \\
\hline & $\begin{array}{l}\text { 5.3 Promover la formación y la colaboración } \\
\text { interprofesional en relación con la valoración y } \\
\text { manejo del dolor en las instituciones } \\
\text { académicas. Nivel de evidencia lb }\end{array}$ & & $\begin{array}{l}\text { Se evidencia } \\
\text { en cuidado de } \\
\text { las } \\
\text { comunidades II } \\
\text { Consulta de } \\
\text { riesgo } \\
\text { cardiovascular } \\
\text { en el dolor } \\
\text { crónico, } \\
\text { interactuar con } \\
\text { el médico del } \\
\text { programa }\end{array}$ \\
\hline
\end{tabular}




\begin{tabular}{c} 
5.4 Los profesionales de salud deben \\
participar en la formación continua para \\
mejorar los conocimientos específicos y las \\
habilidades relacionadas con la valoración y \\
manejo del dolor de forma efectiva basándose \\
en la guía de valoración y manejo del dolor (3 \\
ed). Nivel de evidencia: IV \\
\hline $\begin{array}{c}\text { 6.1 Establecer la valoración y manejo del } \\
\text { dolor como una prioridad estratégica en salud. } \\
\text { Nivel de evidencia IV }\end{array}$ \\
$\begin{array}{c}\text { 6.2 Establecer un modelo de cuidados para } \\
\text { apoyar la colaboración interprofesional para la } \\
\text { valoración y manejo del dolor eficaz. Nivel de } \\
\text { evidencia Ilb }\end{array}$ \\
$\begin{array}{c}\text { 6.3 Utilizar el proceso de transferencia del } \\
\text { conocimiento y las estrategias multimodales } \\
\text { en las instituciones para ayudar a los } \\
\text { profesionales de la salud a utilizar en la } \\
\text { práctica la mejor evidencia en la valoración y } \\
\text { manejo del dolor. Nivel de evidencia III }\end{array}$ \\
$\begin{array}{c}\text { 6.4 Utilizar un enfoque sistemático para toda } \\
\text { la institución para implantar la guía de buenas } \\
\text { prácticas Valoración y manejo del dolor y } \\
\text { proporcionar los recursos y los apoyos } \\
\text { administrativos e institucionales para facilitar } \\
\text { su adopción. Nivel de evidencia IV }\end{array}$ \\
\hline
\end{tabular}

Fuente: Elaboración propia de los autores, año 2016.

Las recomendaciones de planificación no se declaran en ninguno de los componentes de los cursos.

En relación con las recomendaciones de planificación, en el curso de cuidado a las comunidades III hace falta profundizar en el área de formación sobre manejo del dolor. Pero sí se encontró el abordaje a cuidadores, siendo escasa la declaración de temáticas relacionadas con preocupaciones y creencias; por otra parte, en el componente específico se incluyen temáticas relacionadas con el tratamiento farmacológico y no farmacológico del dolor.

Con respecto a la recomendación de evaluación (Revaloración), el abordaje que realiza el curso de comunidades II y III evidencia una revaloración en el tema de dolor crónico en los programas de riesgo cardiovascular, control prenatal y planificación. No se evidencia una revaloración con respecto al dolor agudo en los otros cursos.

En la recomendación de educación el curso de comunidades II declara ofrecer educación para la salud sobre el dolor en situaciones de cronicidad. Con respecto a la recomendación, actualmente, el programa de enfermería tiene previsto implementar la guía de valoración y manejo del dolor en el currículo en forma transversal.

En relación a la recomendación de organización y directrices, actualmente en las guías del programa de enfermería no se evidencian las temáticas de la práctica para mejorar la evidencia en la valoración y manejo del dolor.

Teniendo en cuenta el proceso de levantamiento de los contenidos que se encuentran actualmente declarados en las guías cátedra de la malla curricular del programa de enfermería UNAB, puede evidenciarse que las recomendaciones que más se encuentran descritas son las relacionadas con valoración e implementación de manejo farmacológico y no farmacológico; quedando sin ser declaradas las recomendaciones de planificación a través de un plan integral de cuidados y la recomendación de volver a valorar la respuesta de la persona a las intervenciones de manejo de dolor.

El currículo del programa de enfermería actualmente enseña a través del curso de Investigación II temáticas relacionadas con la práctica basada en la evidencia, donde los estudiantes adquieren conceptos y herramientas que le permiten fortalecer la fundamentación teórica y metodológica de la EBE lo cual se espera contribuya a un mejor desempeño profesional.

En general, una vez realizado el levantamiento de los contenidos curriculares según las recomendaciones se lograron identificar los mínimos contenidos curriculares a garantizar tales como: mecanismos del dolor, clasificación, intervenciones farmacológicas, no farmacológicas, 


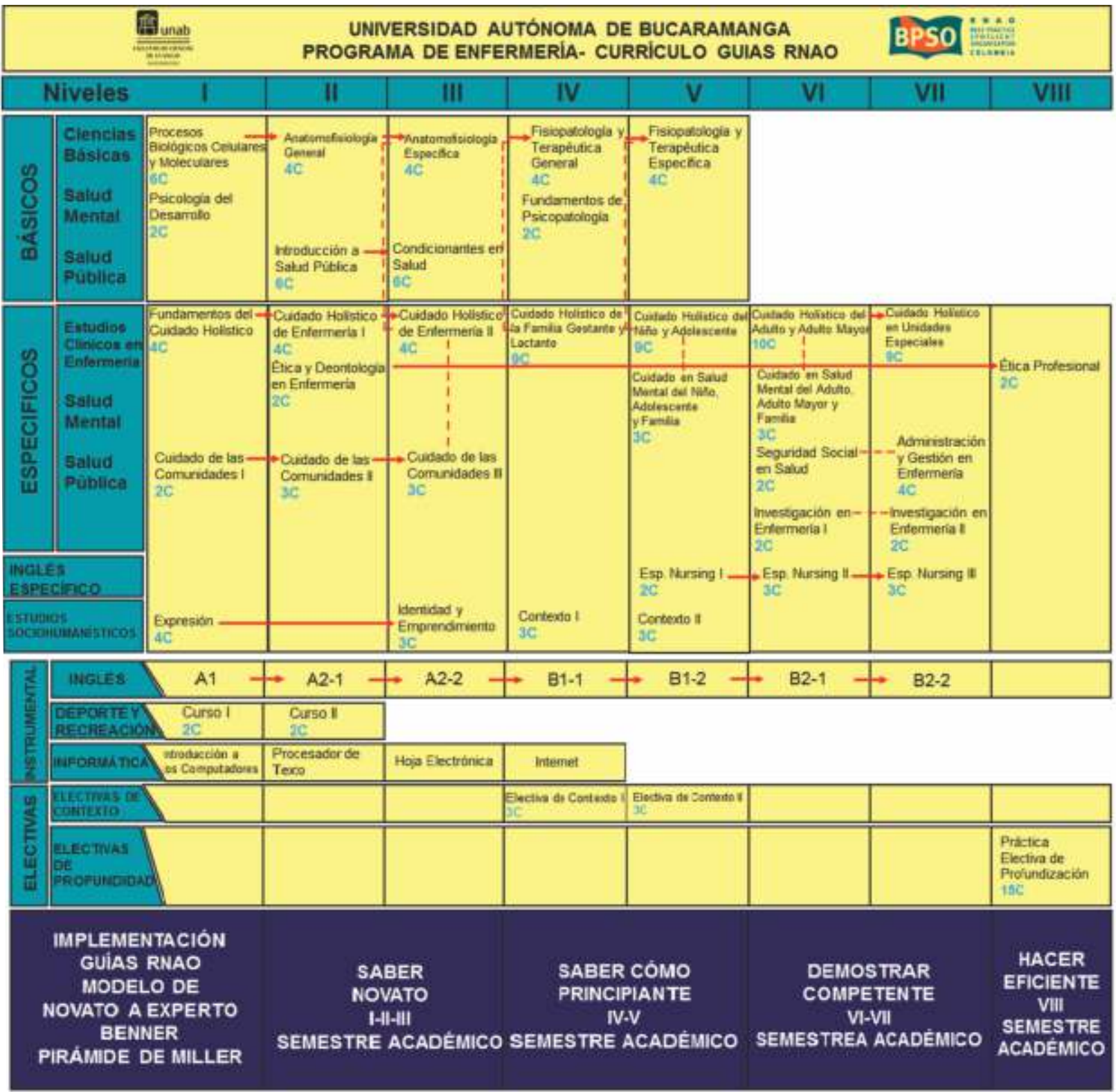

Fuente: Documentos programa de Enfermería 2017

Figura 2. Malla curricular con los componentes del programa de Enfermería UNAB.

implicaciones éticas, gestión y fortalecimiento de una la actitud profesional que permita brindar un plan de atención integral del ser humano en cada una de las etapas del ciclo vital.

Además, se generaron discusiones académicas respecto a las temáticas que deben incluirse según recomendaciones en los diferentes componentes de la malla curricular del programa de enfermería de la UNAB, teniendo en cuenta hacer énfasis en las recomendaciones de planificación, es importante identificar las metas del paciente y su familia para el manejo del dolor y las estrategias adecuadas para garantizar un enfoque integral así como establecer objetivos claros que involucren no solo al paciente sino al equipo interprofesional. En la recomendación de valoración es importante volver a valorar la respuesta de la persona a las intervenciones de manejo de dolor constantemente utilizando la misma herramienta de valoración. Con cada una de estas recomendación los docentes líderes de los diferentes cursos del componente básico, específico y de profundización fueron responsables de facilitar los contenidos temáticos a través de la plataforma TEMA con estrategias que incluyen las TIC para de esta forma motivar la participación activa y la apropiación del conocimiento por parte de los estudiantes; así mismo deben extenderse estos contenidos temáticos con evidencia científica en el contexto de las prácticas formativas en cada uno de los cursos de cuidado holístico. 
En este momento el proceso evaluativo está siendo monitoreado a través de preguntas en las diferentes pruebas orales y escritas del curso así como en la evidencia de la implementación de los planes de mejoramiento en el cuidado integral al paciente, familia o colectivo que se desarrollan a través de los diferentes seminarios del núcleo integrador de los cursos de cuidado holístico y en las prácticas de profundización de último semestre, cuyos resultados se esperan poder publicar en un futuro artículo.

Paralelo a este proceso se organizó un proyecto de investigación que permitiera generar un instrumento que evaluara el nivel de conocimientos respecto a la valoración y manejo del dolor. Dicho instrumento se denominó conocimientos sobre valoración y manejo del dolor basados en la evidencia científica (CVMD-BE): Instrumento de elaboración propia, se elaboró teniendo en cuenta las recomendaciones de la guía de buenas prácticas clínicas, valoración y manejo del dolor RNAO (Versión 2013). Las recomendaciones tienen que ver con tres grandes componentes: práctica, formación del recurso humano y organizaciones de salud; por este motivo se crearon tres cuestionarios como se desglosa a continuación:

- Cuestionario relacionado con la Práctica Clínica (total 31 preguntas); consta de las dimensiones conocimientos en valoración (10 preguntas), planeación (6 preguntas), implementación (9 preguntas) y revaloración (6 preguntas).

Las preguntas son de falso y verdadero, donde las opciones de falso obtienen el valor de 1 y verdadero el valor de 2. A mayor puntuación mayor nivel de conocimientos.

- Cuestionario apéndice en Formación del Recurso Humano (12 ítems). Se construyó un apéndice con preguntas que buscan determinar las condiciones de las instituciones formadoras del recurso humano (universidades) para la implementación de guías en valoración y manejo del dolor en el currículo.

- Cuestionario apéndice en Organizaciones de Salud (14 preguntas). Se construyó un apéndice con preguntas que buscan determinar las condiciones de las instituciones de salud para la implementación de guías en valoración y manejo del dolor.

También se realizó la validación cultural del instrumento titulado Attitudes Survey Regarding Pain" (Ferrell and McCaffery) (NKASRP) o conocimientos y actitudes respecto al dolor, que se desarrolló en 1987, consta de El NKASRP tiene 22 ítems de categoría verdadero/falso, 14 de opción múltiple con única respuesta y dos casos estudios con dos preguntas cada una para un total de 38. Se realizó la validación en el contexto Colombiano haciendo uso de la metodología de validación lingüística ajustada, donde participaran personas formadas en lengua (castellana e inglés), entrevistas cognoscitivas a población diana y retroalimentación de los autores originales para la versión definitiva a aplicar en el estudio propuesto. Este instrumento consta de 38 preguntas. Se realizó la búsqueda del instrumento de origen inglés, el cual ha sido validado en contexto mexicano. Se tuvo en cuenta las dos versiones para generar el proceso de validación cultural. Ya se cuenta con la traducción directa y las entrevistas cognoscitivas.

Este paquete de instrumentos se aplicó a estudiantes de enfermería de los semestres de 7 y 8 de la UNAB. Esto con el fin de tener una línea de base antes de la implementación, que se adelanta a estudiantes de nueva cohorte.

Otra de las fortalezas es contar con tres sitios de práctica que han venido trabajando con el enfoque de implementación de guías de la RNAO entre ellas FOSCAL, FOSCAL Internacional y Fundación Cardioinfantil-IC en Bogotá.

\section{Conclusiones}

Esta primera fase de implementación de la Guía de Buenas Prácticas Clínicas de la valoración y manejo del dolor de RNAO, ha permitido un trabajo en equipo dinámico, constructivo y de empoderamiento del conocimiento por parte de los docentes del Programa de Enfermería UNAB.

El proceso de levantamiento de información a través de la revisión de las guías cátedra de cada uno de los cursos de la malla curricular del programa de enfermería permitió identificar los vacíos y las acciones de implementación a tener en cuenta para incluir en los contenidos curriculares respecto al uso de recomendaciones de práctica clínica para la valoración y manejo del dolor, acciones que a futuro se espera que continúen posicionando la profesión con altos estándares de calidad en la atención humanizada y con evidencia científica en el currículo del programa de enfermería de la UNAB.

El grupo de docentes del programa de enfermería logró reconocer la importancia que tiene la implementación de la guía de buenas prácticas clínicas en la valoración y manejo del dolor de RNAO de forma transversal en el currículo, así como tener una herramienta de validación de las competencias del estudiante en el conocer de los mecanismos del dolor, sus diferentes clasificaciones, las intervenciones farmacológicas y no farmacológicas, sus implicaciones éticas, la gestión y el fortalecimiento de una actitud profesional que permita brindar un plan de atención integral del ser humano en cada una de las etapas del ciclo vital.

Se evidencia una articulación entre la teoría, la práctica y la EBE al conjugarse la rigurosidad científica y la experiencia profesional del equipo de la RNAO con el compromiso, responsabilidad y calidad académica del programa de enfermería de la UNAB, en el recorrido llevado a cabo en el proceso de implementación de la guía de Guía de Buenas Prácticas Clínicas de la valoración y manejo del dolor de RNAO. 
Contar con instrumentos que permitan identificar el nivel de conocimientos alrededor de la valoración y manejo del dolor son herramientas fundamentales para identificar líneas de base y en el tiempo valorar los cambios en la implementación.

\section{Conflicto de intereses}

Los autores manifiestan no tener conflicto de interés.

\section{Referencias}

1. Castro J, CM Benavides C. En Dolor, el quinto signo vital. Universidad del Rosario. [Internet]. 2005. [Acceso 23 noviembre 2016]. Disponible en: http://www. urosario.edu.co/Universidad-Ciencia-Desarrollo/ur/ Fasciculos-Anteriores/Tomo-I---2006/Fasciculo8/pdf/2006_fa08_Dolor_el_quinto_signo_vital /

2. Ministerio de la Protección Social y Universidad de Antioquia, Facultad Nacional de Salud Pública. Bogotá. Análisis de la situación de salud en Colombia, 2002-2007. [Internet]. 2010. [Acceso 20 diciembre 2016]. Disponible en: https://www.minsalud.gov.co/Documentos $\% 20 y \% 20$ Publicaciones/ASIS-Tomo\%20III--

3. Babarro, Alberto Alonso. La importancia de evaluar adecuadamente el dolor. Atención primaria. [Internet]. 2011. [Acceso 20 diciembre 2016]; 43 (11), 568-575. Disponible en: http://www.sciencedirect.com/science/ article/pii/S0212656711000412

4. Herdman T.H, Kamitsuru S. Diagnósticos enfermeros: definiciones y clasificación 2015-2017. Primera edición. Elsevier.

5. Internacional Association for the study of pain. Disponible en:http://www.iasp-pain.org/Education/ Content.aspx? ItemNumber=1698

6. Palanca I; et al. Grupo de Expertos. Unidad de tratamiento del dolor: estándares y recomendaciones de calidad y seguridad. Madrid: Ministerio de Sanidad, Política social e igualdad. 2011.

7. Motov, Sergey, Khan, Abu. Problems and barriers of pain management in the emergency department ¿Are we ever going to get better?. In: Journal of Pain Research. 2009; 2: 5-11.

8. Ministerio de la Protección Social. Análisis de la situación de salud en Colombia de 2002 a 2007. Tomo III: morbilidad y mortalidad de la población Colombiana. Disponible en: http://www.minsalud.gov.co/ Documentos $\% 20 y \% 20$ Publicaciones/ASIS-Tomo\%20III-Morbilidad\%20y\%20mortalidad\%20de\%20la\%20poblaci $\%$ C3\%B3n\%20colombiana.pdf

9. Miguel-Martín M, Losa M, Silva do Rosario T, Salvadores P, Gómez-Arnáu JI, Díaz-Cañabate, et al. Grado de conocimientos y actitudes de la enfermería de la Comunidad Autónoma de Madrid (CAM) ante el dolor: diplomados en enfermería y profesionales. Rev. Soc. Esp. Dolor [Internet]. 2012 [citado 2015 ene 23]; 19(6): 293300. Disponible en: http://scielo.isciii.es/ scielo.php? $\mathrm{s} c \mathrm{ript}=\mathrm{s}$ c i a r t e $\times$ t \& p i d = S 1134 $804620120006000 \overline{0} 3 \&$ Ing $=$ es.

10. Registered Nurses' Association of Ontario. Assessment and Management of Pain (3rd ed.). Toronto, Canada: Registered Nurses' Association of Ontario.2013.
Disponible en: http://rnao.ca/sites/rnao-ca/files/Assess AndManagementOfPain_15_WEB_FINAL_DEC_2.pdf

11. Borré YM, Vega Y. Calidād Percibida De La Atención De Enfermería Por Pacientes Hospitalizados. Cienc. enferm. [Internet]. 2014 [citado 2016 Jul 09]; 20 (3): 81-94. Disponible en: http://www.scielo.cl/scielo.php?script= sci_arttext\&pid=S0717-95532014000300008\&lng=es

12. Montealegre G, Doris C. Retos para enfermería en el cuidado de personas con dolor: una forma de humanización. Revista CUIDARTE [Internet]. 2014; 5(1). Disponible en: https://www.revistacuidarte.org/index.php/ cuidarte/article/view/106

13. Brennan F, Cousins MJ. El alivio del dolor como un derecho humano. Rev. Soc. Esp. Dolor [Internet]. 2005 [citado 2017 oct 25]; 12(1): 17-23. Disponible en: http://scielo.isciii.es/scielo.php?script=sci_arttext\&pid=S1 134-80462005000100004\&lng=es.

14. García M, Hernández Y, Gomez B, García A. Calidad del cuidado de enfermería en pacientes hospitalizados con dolor. Revista Horizontes [Internet]. 2011: 55-69.

15. Cosín MT, Quintana CO, García AM. Enfermería Basada en la Evidencia. Orígenes y fundamentos para una práctica enfermera basada en la evidencia. Nure investigación; 52: 1-7.

16. Proyecto Educativo del Programa de Enfermería. Universidad Autónoma de Bucaramanga. 2016.

17. Zavala-Rodríguez MR, González-Salinas JF. Conocimiento y manejo del dolor en alumnos de enfermería. Aquichán [Internet]. 2008 [citado 2016 septiembre 09]; 8(2): 212-226. Disponible en: http://www.scielo.org.co/scielo.php?script=sci_arttext\&pid $=$ = $1657-59972008000200009 \&$ Ing=en

18. Registered Nurses' Association of Ontario. Best Practice Spotlight Organizations (BPSO). Disponible en: http://rnao.ca/bpg/bpso.

19. Montealegre D. Manejo del dolor: un reto permanente para enfermería. En: Cuidado y práctica de Enfermería. 2000: 197-203.

20. Rodríguez VA, Paravic TM. Enfermería basada en la evidencia y gestión del cuidado. Enferm. glob. [Internet]. 2011 [citado 2016 mayo 10]; 10(24). Disponible en: http://dx.doi.org/10.4321/S1695-61412011000400020

21. Falconí C, Britto CJ, Verkovitch I. Integración de la enseñanza de la práctica de enfermería basada en la evidencia científica. Aquichan. 2015; 15 (4): 541-553. Disponible en: https://doi.org/10.5294/aqui.2015.15.4.9.

22. Vásquez M. La Enfermería Basada en la Evidencia y la gestión del cuidado. Rev Univ. salud [Internet]. 2014 [citado 2016 July 10]; 16(1): 05-08. Disponible en: http://www.scielo.org.co/scielo.php?script=sci_arttext\&pid $=$ S0124-71072014000100001\&lng=en.

23. República de Colombia. Ley 266 de 1996 de Enero 25. Por la cual se reglamenta la profesión de enfermería en Colombia y se dictan otras disposiciones. Boletín Oficial del Estado, n 42.710, (05-02-1996).

24. Plan de desarrollo institucional (2013 a 2018). Universidad Autónoma de Bucaramanga.

25. Registered Nurses Association of Ontario [Internet]. Toronto: Best Practice Spotlight Organization (BPOS) Impact Survey Summary of Survey Results; 2011 [Access 2016 April 13]. Disponible en: http://rnao.ca/bpg/bpos/ impact_survey

26. Registered Nurses' Association of Ontario. Herramienta de implantación de buenas prácticas. Guía de Buenas Prácticas. 2nd ed. Toronto, Canadá: Registered Nurses Association of Ontario. 2012. Disponible en: http://rnao.ca/sites/rnao-ca/files/Toolkit_en15.pdf 